\title{
Current Paradigms to Explore the Gut Microbiota Linkage to Neurological Disorders
}

The investigation of gut microbiota in neurological disorders is a very exciting field of future neurology. My Editor's Pick for this year's issue is by Sharma et al. This paper details what is known about the link between gut dysbiosis and neurological disorders and provides examples that showcase this. Understanding the interactions between microbiota and EDITOR'S

the brain may open up a new avenue of therapeutic approaches for hard-to-treat neurological disorders. We hope you enjoy reading this timely article.

Authors:

Disclosure:

Acknowledgements:

Received:

Accepted:

Keywords:

Citation:
Varruchi Sharma, ${ }^{1}$ Atul Sankhyan, ${ }^{2}$ Anshika Varshney, ${ }^{3}$ Renuka Choudhary, ${ }^{*}$ Anil K. Sharma ${ }^{3}$

1. Department of Biotechnology, Sri Guru Gobind Singh College, Chandigarh, India

2. Swami Devi Dyal Group of Professional Institutes, Panchkula, India

3. Department of Biotechnology, Maharishi Markandeshwar University, Haryana, India *Correspondence to anibiotech18@gmail.com

The authors have declared no conflicts of interest.

The authors would like to thank Maharishi Markandeshwar University, Haryana, India, for providing the requisite facilities to carry out this study.

14.03 .20

17.04.20

Gut microbiome, homeostasis, modulation, neurological disorders, therapeutic intervention.

EMJ Neurol. 2020;8[1]:68-79.

\section{Abstract}

It has been suggested that an intricate communication link exists between the gut microbiota and the brain and its ability to modulate behaviour of an individual governing homeostasis. Metabolic activity of the microbiota is considered to be relatively constant in healthy individuals, despite differences in the composition of microbiota. The metabolites produced by gut microbiota and their homeostatic balance is often perturbed as a result of neurological complications. Therefore, it is of paramount importance to explore the link between gut microbiota and brain function and behaviour through neural, endocrine, and immune pathways. This current review focusses on the impact of altered gut microbiota on brain functions and how microbiome modulation by use of probiotics, prebiotics, and synbiotics might prove beneficial in the prevention and/or treatment of neurological disorders. It is important to carefully understand the complex mechanisms underlying the gut-brain axis so as to use the gut microbiota as a therapeutic intervention strategy for neurological disorders. 


\section{INTRODUCTION}

The human body harbours a diverse community of symbiotic, commensal, and pathogenic micro-organisms including bacteria, viruses, and fungi, collectively known as the microbiota. In recent times, the relationship between brain and gut microbiota has become an important component of wider research studies. The microbiome consists of the combined genetic material of the micro-organisms in a particular environment that may constitute the whole body or a specific part of the body. There exists a bidirectional microbiome gut-brain axis communication, which enables the gut microbiota to interact with the brain and vice versa. ${ }^{1}$ This communication between the microbiota and central nervous system (CNS) is established through autonomous, neuroendocrinological, gastrointestinal, and immunological systems. Pathological complications are known to alter the levels of neurotransmitters, and therefore the balance between them, exacerbating the hypothalamuspituitary-adrenal (HPA) axis activity and leading to chronic inflammation. ${ }^{2}$ The intestinal secretions, porousness of the gut, and gut motility, along with the structure-function activity relationship, are also greatly influenced by the CNS. Conversely, the gut microbiota, by secreting certain chemicals, are known to affect the brain functions as well. ${ }^{2}$ Even anxiety/stress and social issues are reported to affect bile secretions by altering the genes responsible for bile production. ${ }^{2}$

Many preclinical studies have suggested that the microbiome and the microbiota could be the key regulators in predisposing organisms towards various disorders, including neurodegenerative disorders, because alterations to the composition of the human gut microbiota are linked to a variety of neuropsychiatric conditions including depression, autism, Alzheimer's disease (AD), multiple sclerosis (MS), and Parkinson's disease (PD). However, studies are limited and it is not confirmed whether there is a direct link between the microbiota and brain disorders, or if microbiota indirectly influence by virtue of secondary effects. ${ }^{3}$ It has been shown that one may improve the gut microbiota by the intake of probiotics, fermented foods, and prebiotic fibres in the diet; a healthy lifestyle; reducing sugar consumption; avoiding antibiotic use; and decreasing stress.

There are a number of mechanisms that support the interaction between the brain and gut microbiota, either in relation to neural, endocrine, immune, or metabolic signalling pathways. There is also a significant bridging between the brain and gut microbiota at the chemical level, as has been seen through the use of various hormones such as corticotropin-releasing hormone in the pituitary and the neurotransmitters dopamine, serotonin, and GABA. ${ }^{3}$

\section{GUT MICROBIOTA-BRAIN CROSS TALK}

Gut microbiota are thought to be greatly influenced by stimulating the HPA axis and sympathetic nervous system. The stimulation of the HPA axis is a major constituent of stress response, which has been well established through genetic and post-delivery environmental factors. ${ }^{4}$ Past studies in mouse models have observed variations in host behaviour in the stress stimuli response. The observations indicated that the gut microbiota can influence the progression of the HPA reaction to stressors. ${ }^{5}$

Significant behavioural alterations have been known to be caused by a diverse range of micro-organisms. In mice, toxoplasmosis and bacterial infections altered the behaviour of the mice in such a way that they became unresponsive to the smell of cats, meaning the mice could be easily preyed upon. ${ }^{6}$ There are some probable pathways involved in the association between the gut microbiota and brain that could help the transmission of information from gut to brain. The information can be processed for better understanding through the roles of short-chain fatty acids (SCFA) and neuroglia, and tryptophan and its metabolites, as well as accessing pathways at the neural level. ${ }^{7}$ At neural levels, the conduction of information from the intestine to the CNS through nerves/neurons, has been successfully demonstrated in mice. ${ }^{8}$ In one study, Bifidobacterium infantis administration to germ-free mice resulted in increased expression of $c$-Fos, ${ }^{9}$ a principal alteration in the brain that changes the conversion of shortterm incitements into long-term responses. The enhanced expression was moderately 
repressed by treatment with capsaicin. ${ }^{10}$ It was observed that the serotonin released from enterochromaffin-like cells, which originate in the gastric gland, act upon serotonin Type 3 receptors, and lead to the transfer of information engendered in the gut to the brain. . $^{511}$

\section{SHORT-CHAIN FATTY ACIDS AND CHANGES IN MICROGLIA}

Dietary fibres, upon absorption in the gut, have been reported to yield SCFA. These SCFA have been shown to be absorbed through the colon, and are used as an energy source. More recently, some receptors have been identified which has led to the novel, functional characterisation of SCFA. Butyric acid and its impact on the CNS has been observed previously in animal studies, because it functions as an antidepressant. ${ }^{12}$

\section{Tryptophan and its Metabolites}

Tryptophan is an essential amino acid found in the digestive system. It acts as a precursor in serotonin biosynthesis, blood platelets biosynthesis, and for numerous molecules in the CNS.13 The kynurenine pathway is known to be controlled by the enzyme indolamine 2, 3-dioxygenase, in which the majority of tryptophan is involved in. Further, the cytokine levels reportedly boost the tryptophankynurenine route in the pathway. The metabolic products of kynurenine have been reported to affect the functioning of neural cells. ${ }^{14}$ Kynurenic acid (KYNA), produced by kynurenine, acts antagonistically at the broad-spectrum ionotropic glutamate receptors. In case of neurological complications such as schizophrenia, the levels of this tryptophan metabolite are elevated in the brain and cerebrospinal fluid. KYNA is known to antagonise the function of a7 nicotinic and $\mathrm{N}$-methyl-d-aspartate (NMDA) receptors, both of which are required for cognitive processes and normal brain development. Therefore, KYNA levels are very critical for the predisposition towards neurological disease. ${ }^{15}$

Similarly, carbohydrates levels are thought to influence the mood and behaviour of humans. Carbohydrates in our diet are known to stimulate insulin discharge in the body; they lead to the release of blood sugar into cells and the production of energy, simultaneously triggering the entry of tryptophan into the brain which has a pronounced effect on neurotransmitters levels. As the production of serotonin and tryptophan in the brain aid mental wellbeing, some studies suggest a diet low in carbohydrates to alleviate depression. ${ }^{16}$

\section{FUNCTIONAL ASPECTS OF GUT MICROBIOTA}

The human microbiome is composed of viruses, bacteria, protozoa, and fungi and the interaction network of these microbiota plays an essential role in overall health. In humans, the microbes are present externally, on the skin, mouth, intestine, etc., and internally, in the digestive system, lungs, etc. ${ }^{17}$ Bacteria are the most predominant members of the microbiome community; they are highly dense in the gut, predominantly the colon. Firmicutes and Bacteroidetes are the most abundant phyla and are major constituents in functioning with reference to the microbiome, while Proteobacteria, Verrucomicrobia, Actinobacteria, and Fusobacteria are reportedly present at lower levels. ${ }^{18}$ All the microbes associated with the gut microbiome have a vast range of functions with respect to the human body as they aid digestion, develop the immune system, and support mental health. ${ }^{19}$

The key communication route between CNS and gut microbiota is via the vagus nerve, which acts as a thread between them. Fluctuation in the signal of the nerve can result in immunoregulation effects and dysfunction of the nervous system. This may lead to gastrointestinal diseases, intestinal distress, anxiety, depression, diseases such as irritable bowel syndrome, MS, AD, PD, and inflammatory bowel disease (IBD), etc. ${ }^{20}$ Several studies have evidenced the improvement in levels of cytokines by vagal stimulation. ${ }^{21,22}$ Enteroendocrine cells transmit biological signals from the gut to the brain via stimulation by bacterial metabolites, hormones such as cholecystokinin, peptide YY, and serotonin. These mediators bind to chemoreceptors and upon binding, activate the neural afferent fibres. In another study, subtypes of enteroendocrine cells were reported to complete the signal by direct communication with the gut through vagal afferent fibres. ${ }^{23}$ 
Table 1: Some of the major disorders associated with altered gut microbiota.

\begin{tabular}{|c|c|c|c|}
\hline Disease associated & Disease type/subtype & Characteristics & Reference \\
\hline Alzheimer's disease & $\begin{array}{l}\text { Chronic and irreversible } \\
\text { neurodegenerative disease }\end{array}$ & $\begin{array}{l}\text { Central nervous system } \\
\text { dysfunction }\end{array}$ & \multirow[t]{3}{*}{$24-26$} \\
\hline Multiple sclerosis & Inflammatory disease & $\begin{array}{l}\text { Immune-mediated } \\
\text { demyelination of the neural } \\
\text { axon }\end{array}$ & \\
\hline Parkinson's disease & Neurodegenerative disorder & $\begin{array}{l}\text { Multifactorial motor } \\
\text { symptoms }\end{array}$ & \\
\hline \multirow[t]{5}{*}{ IBS } & Bowel disorder & $\begin{array}{l}\text { Abdominal discomfort, } \\
\text { adaptations in bowel habits }\end{array}$ & \multirow[t]{5}{*}{27} \\
\hline & IBS with diarrhoea & $\begin{array}{l}\text { Loose, frequent stools; } \\
\text { abdominal pain; and } \\
\text { uneasiness, gastric, etc }\end{array}$ & \\
\hline & IBS with constipation & $\begin{array}{l}\text { Chronic or persistent } \\
\text { constipation }\end{array}$ & \\
\hline & Mixed or cycling & $\begin{array}{l}\text { Digestive complications: } \\
\text { cramping, belly pain, and } \\
\text { bloating }\end{array}$ & \\
\hline & Unsubtyped IBS & $\begin{array}{l}\text { Problems associated to } \\
\text { motion/movement through } \\
\text { the gastrointestinal tract }\end{array}$ & \\
\hline \multirow[t]{2}{*}{ Inflammatory bowel disease } & Bowel disease & $\begin{array}{l}\text { Inflammation in the gut } \\
\text { subsequent to the grouping } \\
\text { of environmental and } \\
\text { inherited factors }\end{array}$ & \multirow[t]{2}{*}{28,29} \\
\hline & Paediatric Crohn's disease & $\begin{array}{l}\text { Enlarged profusions } \\
\text { of Enterobacteriaceae, } \\
\text { Pasteurellaceae, } \\
\text { Fusobacteriaceae, } \\
\text { Neisseriaceae, } \\
\text { Veillonellaceae, } \\
\text { and Gemellaceae, } \\
\text { reduced profusions } \\
\text { of Bifidobacteriaceae, } \\
\text { Erysipelotrichaceae, } \\
\text { Clostridiales, and } \\
\text { Bacteroidales }\end{array}$ & \\
\hline Cardiovascular disease & Heart disease & $\begin{array}{l}\text { Microbiota-dependent } \\
\text { pathway }\end{array}$ & 29 \\
\hline
\end{tabular}

IBS: irritable bowel syndrome.

\section{DISORDERS ASSOCIATED WITH ALTERED GUT MICROBIOTA}

The CNS is sensitive to microbiota changes occurring through numerous pathways, which has an important role in the progression of neurological disorders (Table 1). ${ }^{24-29}$ Human Type I IFN, NF-kB, and the inflammasome are the major components associated with the above pathways and play an important role in influencing the microbiota. ${ }^{29}$ 


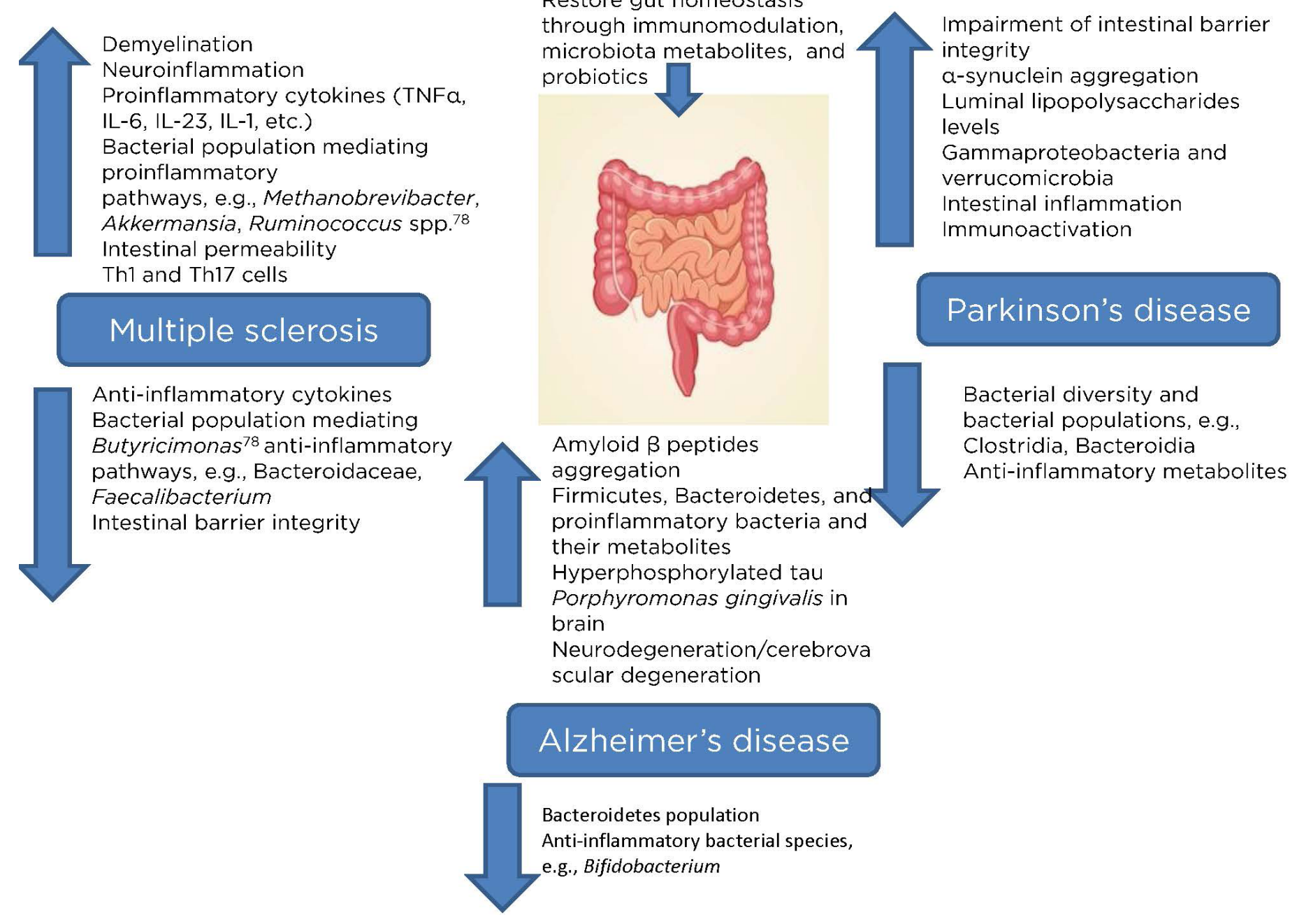

Therapeutic intervention Restore gut homeostasis through immunomodulation, microbiota metabolites, and probiotics

Amyloid $\beta$ peptides

aggregation

Firmicutes, Bacteroidetes, and their metabolites Hyperphosphorylated tau Porphyromonas gingivalis in rain

Neurodegeneration/cerebrova cular degeneration

\section{Alzheimer's disease}

Figure 1: Microbiota dysbiosis due to pathological complications of major neurological disorders (multiple sclerosis, Alzheimer's disease, and Parkinson's disease) providing a therapeutic window via restoring microbiota homeostasis.

\section{Multiple Sclerosis}

MS is an immune-mediated inflammatory ailment in which myelin loss results in neurological complications, including damages at the visual, motor, sensual, autonomous, and cognitive level. There are a variety of general factors reported to be associated with MS that are responsible for damage to the insulating covers of the nerve cells in the brain and spinal cord, resulting in physical, neurological, and sometimes psychiatric problems (Figure 1). ${ }^{24}$

The gut microbiome is said to play a vital role in the development of MS, as evidenced by the differences in the specific gut microbial taxa present in patients with this autoimmune disease in comparison to healthy individuals. ${ }^{25}$
Autoimmunity is known to have direct control over the response of the immune system towards the gut microbiota, thus altering the overall composition of the resident microbiota. ${ }^{26}$ Significant microbial alterations in the gut, as a result of the presence of the major microbial population associated with proinflammatory pathways, enhance the pathogenesis of MS. Gut microbial dysbiosis further increases the intestinal permeability, microbial translocation, and local and systemic inflammation, resulting in MS. Additionally, neuroinflammation and demyelination are involved, along with a significant impact on the natural killer $\mathrm{T}$ cell population. ${ }^{27}$ Firmicutes and Bacteroidetes populations, as well as the class of alphaproteobacteria, were reportedly reduced as a result of the disease, while there was an increase in the 
class of gammaproteobacteria. ${ }^{28}$ Therefore, the interaction between the gut microbiota and MS appears bidirectional, providing itself a potential therapeutic window for early intervention with probiotics or metabolites produced from the microbiota. ${ }^{30,31}$ Faecal microbiota transplantation (FMT) has been recently utilised, wherein the host aberrant gut microbiome could be replenished in order to replace the aberrant microbiome with a healthy one..$^{32-34} \mathrm{~A}$ case study demonstrated the effectiveness of FMT in a 61-year-old patient with secondary, progressive MS with a history of several episodes of enterocolitis. ${ }^{26,35}$ The Expanded Disability Status Scale (EDSS) of the patient was stabilised, but FMT had limited efficacy in this case..$^{26,36}$

There are several environmental factors that play an important role in the pathogenesis of MS including geographical location, smoking, vitamin A and D, retinol, sodium, body mass, and obesity. At higher latitudes, the difference in sunlight exposure may lead to a decrease in vitamin $D$ levels, ${ }^{37}$ which is significant because vitamin $D$ has been reported to prompt anti-inflammatory immune cells. ${ }^{38}$ Disease risk is doubled by smoking because of the higher levels of cotinine. ${ }^{39}$ Another study disclosed that the immune-regulatory characteristics of vitamin A might be pertinent to MS because the transitional levels of retinol binding protein have an association with lower incidence of disease risk. ${ }^{40,41}$

Plasma cells of the gut and IgA antibodies were reportedly found to migrate to the CNS, resulting in an anti-inflammatory effect as observed in a mouse model and in human patients with MS. ${ }^{41}$ MS is known to be driven by $B$ and $T$ cells, and it was found in one study that drugs targeting $B$ cells may alleviate MS. ${ }^{41}$ The same study demonstrated that IgA-producing B cells can move from the gut to the brain, paving a way for novel treatments for MS and other related neurological disorders. ${ }^{42}$

\section{Alzheimer's Disease}

$A D$ is a neurodegenerative, progressive disease caused by CNS dysfunction, which may be described as an advanced death of brain cells that takes place over time and results in excessive neuroinflammation. Memory loss and cognitive decline, along with loss of vision, confusion, depression, anxiety, and fearfulness are the symptoms associated with the disease. One of the key factors attributed to the cause of disease appears to be gut microbiota dysbiosis, as a decrease in microbial diversity in the gut microbiota is observed alongside an increase in profusion of Bacteroidetes (Figure 1). ${ }^{43}$ Dysbiosis, neural loss, and damages to synaptic function, along with the accumulation of tau protein and amyloid- $\beta(A \beta)$ in excess, may lead to neuronal cellular apoptosis in AD. ${ }^{44}$ More recently, the role of the gut microbiome was considered as a dynamic factor in the aetiology of the disease because metabolites derived from microbiota in the cerebral spinal fluid of patients with $A D$ were recognised. ${ }^{45}$

The gut microbiome is a rich source of amyloids, which are present on the surface of bacterial cells. Amyloid fibres can be accumulated, leading to unrestricted immune system activation. Treatment with bacterial amyloids (curli-producing Escherichia coli) has been shown to exhibit amplified neuronal a-synuclein accumulation at both the gut and the brain level. ${ }^{46}$ In another study, the use of injectable bacterial lipopolysaccharide into the brain demonstrated the occurrence of numerous inflammatory features observed in AD. ${ }^{47}$ Microbiota have also been shown to have a therapeutic role in some tumour types: Bifidobacterium, a member of the bifidobacteria species, was shown to be an antitumour agent in a mouse model where the oral administration of Bifidobacterium eliminated the tumour consequence. ${ }^{48}$

An amyloid precursor protein that produced an $A \beta$ peptide lacking glutamic acid at position 22 (E693 $\triangle$ Osaka mutation) was found to be associated with $A D$ through the excessive oligomerisation of the $A \beta .{ }^{49}$ Similarly, the microtubule-associated tau protein is known to keep microtubules straight and strong in a healthy human brain. In $A D$, it was found that the microtubules were no longer able to sustain the transport of nutrients and other essential substances in the nerve cells, which eventually led to cell death. ${ }^{50}$ The hyperphosphorylation of the tau protein detached it from the microtubules and it combined with other associated fragments, resulting in the accumulation of $A B$ plaques, leading to neurodegeneration and AD. ${ }^{51}$ 


\section{Parkinson's Disease}

$P D$ is a neurodegenerative disorder that disturbs movement. Symptoms of PD include tremors in the limbs, hands, or fingers; rigid muscles; loss of automatic activities; fluctuations in tongue movement; and difficulty in walking, balance, and co-ordination, with the brain cells or neurons being the initial starting point of the disease. ${ }^{52,53}$

There are also many inherent and environmental features that are involved in the commencement of the disease..$^{54}$ There are many alterations in the brain of individuals such as the manifestation of Lewy bodies and unusual clumps of the protein a-synuclein, which are considered microscopic disease causing markers of PD (Figure 1). ${ }^{54}$ Age, sex, heredity, and exposure to toxins (herbicides and pesticides) are some of the risk factors that are associated with the disease. In recent studies, it has been reported that a-synuclein action in the nervous system during the initial stages of disease could be correlated to the digestive complications. ${ }^{55}$ a-synuclein overexpression in the mouse model advocates the significant role of microbiota in the disease progression. On the other hand, a-synuclein overexpression in the germ-free mouse model with antibiotic treatment displayed decreased incidence of PD. ${ }^{56}$

\section{NEUROIMAGING METHODS EMPLOYED FOR DIAGNOSIS}

Neuroimaging refers to the imaging of structural, functional, or pharmacological features of the nervous system. There are various methods that have been employed for neuroimaging.

\section{MRI}

$\mathrm{MRI}$ is an extensively used technique which demonstrates properties of the brain at various levels. There is a voluntary transformation among preclinical and clinical backgrounds in this technique. ${ }^{57}$ The major focus of MRI at the structural level is on the assessment of grey matter in the brain, along with demarcation of structural differences with reference to the patient population. Voxel based morphometry is another technique employed for neuroimaging, which is based upon MRI. ${ }^{58}$ Voxel based morphometry explores the principal differences in the brain along with the assessment of grey matter volumes.

\section{Diffusion Tensor Imaging}

Diffusion tensor imaging is subsequent to diffusion-weighted imaging, wherein structural details at the microscopic level are available in reference to the white matter in vivo. Moreover, the same technique has the capability to enumerate the extent of water diffusion. ${ }^{59}$

\section{Fractional Anisotropy}

Fractional anisotropy helps to access the measure of manoeuvring consistency of water dissemination in the tissues, which aids understanding of the white matter at the structural level. In order to define the white matter tracts, other methods such as fibre tracking and three-dimensional visualisation are used, which help to analyse the different diseases/disorders among different population groups. ${ }^{60}$

\section{NEUROIMAGING TECHNIQUES AT FUNCTIONAL LEVEL}

In reference to imaging and measuring the functional changes, numerous techniques can be employed.

\section{Functional MRI Analysis}

Functional MRI relies upon the blood oxygenation level-dependent (BOLD) analysis. The signals transmitted from BOLD are used for understanding the basics of the neural system in both healthy and dysfunctional brains. ${ }^{61}$ Functional MRI and its task specificity help to measure the alterations in neural activities, which defines the response of the brain to external stimulation (stimulus can be sensory, auditory, visual, etc.). In contrast to the working of the human brain, it has also been reported that the brain is functional beneath resting or relaxed states. The resting state uses the BOLD signal for investigating the natural fluctuations in the brain, along with the brain networks centred on the acknowledged interactions.

\section{Functional Connectivity}

Functional connectivity demonstrates the restingstate networks amongst the brain regions with definite functions. Results have been successfully derived from the resting state networks using statistical methods. 
Table 2: Effect of gut microbiome on human health and factors associated with the improvement of the gut microbiome.

\begin{tabular}{|c|c|c|}
\hline Health effects & General characteristics & Reference \\
\hline Whole body & $\begin{array}{l}\text { Bifidobacteria in a newborn baby aids the digestion of the } \\
\text { sugars in breast milk. } \\
\text { Short-chain fatty acids absorption by bacteria. } \\
\text { Regulatory check of gut bacteria over contaminants or } \\
\text { pathogens and brain functions. }\end{array}$ & \multirow[t]{4}{*}{$61-64$} \\
\hline Cardiovascular & $\begin{array}{l}\text { Gut microbiome assists in promoting 'good' HDL } \\
\text { cholesterol and maintaining triglycerides to healthier } \\
\text { levels. }\end{array}$ & \\
\hline Effects on diabetic complications & $\begin{array}{l}\text { Regulation of blood sugar levels restricting diabetic } \\
\text { complications by the gut microbiome. }\end{array}$ & \\
\hline Neurological effects & $\begin{array}{l}\text { Gut microbiome assists in neurotransmitter secretions in } \\
\text { the brain. }\end{array}$ & \\
\hline \multicolumn{3}{|c|}{ Factors associated with the advancements/building of the gut microbiome } \\
\hline Diet & \multicolumn{2}{|l|}{ Derived benefits and recommendations } \\
\hline Diverse range of foods & $\begin{array}{l}\text { Variations in the food intake. } \\
\text { Diet enriched with diverse microbiota. } \\
\text { Inclusion of vegetables, legumes, beans, and fibrous fruits. }\end{array}$ & \multirow[t]{3}{*}{$65-70$} \\
\hline $\begin{array}{l}\text { Addition of fermented foods in the } \\
\text { diet and reduction in the levels of } \\
\text { marketed sugars in diet }\end{array}$ & $\begin{array}{l}\text { Yogurt, sauerkraut, and kefir, all contain healthy bacteria. } \\
\text { Lactobacilli in diet can reduce disease causing agents. }\end{array}$ & \\
\hline $\begin{array}{l}\text { Addition of prebiotic foods, fibrous } \\
\text { foods in the diet }\end{array}$ & $\begin{array}{l}\text { Some food products such as chicory root, artichokes, } \\
\text { bananas, leeks, asparagus, oats, onions, garlic, and } \\
\text { apples. They should be included in the diet to help in the } \\
\text { stimulation of beneficial bacteria. } \\
\text { The fibrous foods and advantageous carbohydrates like } \\
\text { B-glucan are beneficial for humans. } \\
\text { Gut bacteria assist in digestion of food further reducing } \\
\text { the risk of cancer, diabetes, and other disorders. }\end{array}$ & \\
\hline
\end{tabular}

\section{Default Mode Network}

Default mode network displays high levels of activity throughout the resting state and neutralises during the enactment. ${ }^{62}$

\section{Magnetic Resonance Spectroscopy}

Magnetic resonance spectroscopy provides the neural substrate information of the brain, accessing the levels of choline, creatine, glutamate, glucose, and $\mathrm{N}$-acetyl aspartate.

With magnetic resonance spectroscopy, some key investigations can be performed in cases of neurological or neurosurgical disorders. ${ }^{63,64}$

\section{Brain Iron Deposition Imaging}

Iron is a metal that is fundamental to basic processes such as DNA synthesis, respiration in mitochondria, and oxygen transportation. The paramagnetic nature of iron, as determined through advanced MRI techniques, has led to new insights into the iron testimony in vivo in the human brain. ${ }^{65}$

\section{MICROBIOTA VERSUS DISEASE- GENETIC PREVIEW}

There are variety of factors that have been reported to be associated with the improvement of the gut microbiome that impact the human health (Table 2). ${ }^{61-70}$ 
Disease/disorder may lead to alteration in the microbial composition of the gut microbiota, leading to mutations in genes and resulting in immune-level irregularities. Predisposition at the genetic level has been shown to result in the progression of IBD, which is thought to occur because of polymorphisms in the NOD2 gene that codes for a receptor that recognises causative pathogenic micro-organisms. ${ }^{66}$ Mutations in ATG16L1 and IRGM genes have been found to be associated with Crohn's disease, because of eradication of the intracellular bacteria. Acute inflammatory response was found to be associated with other NOD2 variant genes such as L1007X, R7O2W, G908R, and ATG16L1 T300A. ${ }^{66}$ Similarly, some IL such as IL-10, IL-19, IL-27, IL-1RL1, IL-2RA, IL-12RL2, and IL-18R1 and chemokines CCL2 and CCL7 have been found to impact acute inflammatory response. ${ }^{67}$ In another study, determination of microbial factors were studied in reference to host genetics. DNA extraction and amplification studies were performed using general bacterial primers and terminal restriction fragment length polymorphism fingerprints were generated to establish microbial community profiles similar to those seen in nature. ${ }^{68}$

There are many factors that can affect the microbial flora inhabiting the gut, but recent findings discovered that specific host genetic variants influence an individual towards microbiome dysbiosis. ${ }^{69}$ The host regulates the composition of the gut microbiome through the secretion of $\operatorname{lgA}$, antimicrobial peptides, and microRNA. ${ }^{70}$

Genes encode many proteins that shape the microbiome by appropriately regulating the availability of nutrients, and the level of the immune response generated. The secretion of antimicrobial peptides was found to be decreased with mutations in NOD2, ATG16L1, and LRRK2 genes. ${ }^{71,72}$

The abundance of Lactobacillus spp. is found to decrease because of variants in the CLEC7A and CARD9 genes, resulting in altered activities of dendritic cells and macrophages. Increased abundance of Escherichia spp. and Bacteroides vulgatus alongside a decrease in Faecalibacterium spp. was associated with variants in NOD2.73,74 The increased production of IgG and IgA against commensal microbiota has been associated with impaired ATG16L1 signalling, resulting in a loss of tolerance to intestinal microbes. Polymorphisms in MHC Class /I or $H L A$ genes were seen to affect production of IgA in response to microbes. Defects in mucus production were seen in altered intestinal microbiome, while also increasing susceptibility to colitis. $^{75}$

There was a significant variation in the gut microbiota of healthy individuals with a high genetic risk for IBD. Roseburia intestinalis is one of the 20 most abundant species present in the gut microbiota. The IBD genetic risk score was significantly associated with a decrease in Roseburia in the gut microbiota of patients with IBD. ${ }^{76}$ Increased variants in genes involved in bacterial handling (NOD2, IRGM, ATG16L1, CARD9, and FUT2) have been found to be associated with a decrease in Roseburia spp. Roseburia spp. were specifically seen to colonise the mucins, directing mucosal butyrate production. Butyrate derived from clostridium clusters IV, VIII, and XIVa (to which Roseburia spp. belong to) has been shown to induce T-cell regulators, affecting intestinal inflammation. ${ }^{76}$

\section{CHALLENGES AND LIMITATIONS OF THE MICROBIOTA RESEARCH}

The impact of the microbiota on human health has been significant in guiding us to newer diagnostic and therapeutic approaches to treat neurological disorders (Figure 1); however, concrete clinical studies or large cohorts of randomised trials are lacking. Also, complex intricacies of host microbial interactions, microbial environment, the underpinning human microbiome, and how additive, subtractive, or modulatory strategies influence such interactions, are not completely understood. Additionally, there is a lack of knowledge so far about comprehensive understanding of the rules governing invasion, resilience, and succession in a host-associated microbial environment, which is quintessential for the development of longterm cell-based therapeutics. ${ }^{77}$ The majority of studies pertaining to microbiota have been tested in rodents which are correlated to humans in a generalised fashion, despite having differences in their respective microbiomes. Effective therapeutic studies considering microbiota as a 
biomarker are still to be carried out. Moreover, genetic engineering approaches to enable the modification of a wide range of bacterial hosts, along with the creation of disease-relevant sensors, are still at infancy.

Natural commensals like Bacteroides or Clostridia could have the potential to be developed as microbiota-based therapies, but again, pharmacological and safety issues are the major concerns, which are yet to be addressed. Furthermore, microbiota research has been limited to short-term therapies so far, and longterm cellular therapies and how the engineered microbiota establishes at the target site with respect to time and changing environment are still at large. Newer and advanced genetic engineering techniques are required in order to overcome these challenges.

\section{CONCLUSIONS AND FUTURE PERSPECTIVES}

Healthy humans differ from each other in terms of microbiome composition, but the metabolic activity of the microbiome remains relatively constant. Immune response is affected as a result of active transport or diffusion of metabolites produced in the gut. When perturbations in the microbiome occur as a result of disease/disorder, the immune-homeostasis equilibrium is altered, further impacting the drug-metabolic capacity of the host as well. ${ }^{78,79}$ Metabolites such as neurotransmitters, neuromodulators, and SCFA produced by gut microbiota are known to reduce the secretions of proinflammatory cytokines while inducing T-cell regulators development and IL-10 secretions. Some SCFA may also cross into the CNS. Additionally, the integrity of the blood-brain barrier is disrupted during neuroinflammation through the action of IL-1, IL-6, and TNFa, resulting in neurological complications. ${ }^{80}$ Therefore, the gut microbiota greatly influence the brain function and behaviour through neural, endocrine, and immune pathways (Figure 1). Modulation of the microbiome by use of probiotics, prebiotics, and synbiotics might prove beneficial in the prevention and/or treatment of neurological disorders. To fully harness the influence of gut microbiota for the therapeutic intervention in neurological diseases, future research should improve our understanding of the complex mechanisms underlying the gut-brain axis. Moreover, large-cohort studies are required to investigate the therapeutic potential of longterm modulation of the gut microbiota on brain diseases.

\section{References}

1. Dinan TG, Cryan JF. Brain-gutmicrobiota axis and mental health. Psychosom Med. 2017;79(8):920-6.

2. Bruce-Keller AJ et al. Harnessing gut microbes for mental health: getting from here to there. Biol Psychiatry. 2018;83(3):214-23

3. Li $Q$ et al. The gut microbiota and autism spectrum disorders. Front Cell Neurosci. 2017;11:120.

4. Dinan TG et al. Hypothalamicpituitary-gut axis dysregulation in irritable bowel syndrome: plasma cytokines as a potential biomarker? Gastroenterol. 2006;130(2):304-11.

5. Sudo N. Role of gut microbiota in brain function and stress-related pathology. Biosci Microbiota Food Health. 2019;38(3):75-80.

6. Dubey J, Frenkel J. Experimental toxoplasma infection in mice with strains producing oocysts. Journal Parasitol. 1973:505-12.

7. Dalile $\mathrm{B}$ et al. The role of short- chain fatty acids in microbiotagut-brain communication. Nat Rev Gastroenterol Hepatol. 2019:16(8):46178.

8. Sudo $\mathrm{N}$ et al. Postnatal microbial colonization programs the hypothalamic-pituitary-adrenal system for stress response in mice. Journal Physiol. 2004;558(1):263-75.

9. Herrera DG, Robertson HA. Activation of c-fos in the brain. Prog Neurobiol. 1996;50(2-3):83-107.

10. Basso AS et al. Immune-induced flavor aversion in mice: modification by neonatal capsaicin treatment. Neuroimmunomodulation. 2001;9(2):88-94

11. Li HJ et al. Distinct cellular origins for serotonin-expressing and enterochromaffin-like cells in the gastric corpus. Gastroenterol. 2014;146(3):754-64

12. Morrison DJ, Preston T. Formation of short chain fatty acids by the gut microbiota and their impact on human metabolism. Gut Microbes. 2016;7(3):189-200

13. O'Mahony SM et al. Serotonin, tryptophan metabolism and the brain-gut-microbiome axis. Behav Brain Res. 2015;277:32-48.

14. Gao J et al. Impact of the gut microbiota on intestinal immunity mediated by tryptophan metabolism. Front Cell Infect Microbiol. 2018;8:13.

15. Jenkins TA et al. Influence of tryptophan and serotonin on mood and cognition with a possible role of the gut-brain axis. Nutrients. 2016;8(1):56.

16. Rao TSS et al. Understanding nutrition, depression and mental illnesses. Indian J Psychiatry. 2008;50(2):77-82.

17. Laforest-Lapointe I, Arrieta MC Microbial eukaryotes: a missing link in gut microbiome studies. mSystems. 2018;3(2):e00201-17. 
18. $\mathrm{Yu} Y \mathrm{~N}$ et al. Berberine may rescue Fusobacterium nucleatuminduced colorectal tumorigenesis by modulating the tumor microenvironment. Oncotarget. 2015;6(31):32013.

19. Kumar A, Chordia N. Role of microbes in human health. Appli Microbiol Open Access. 2017;3:2.

20. Cryan JF et al. The gut microbiome in neurological disorders. Lancet Neurol. 2020;19(2):179-94.

21. Wang HX, Wang YP. Gut microbiota-brain axis. Chin Med J. 2016;129(19):2373.

22. Flowers SA, Ellingrod VL. The microbiome in mental health: potential contribution of gut microbiota in disease and pharmacotherapy management. Pharmacotherapy. 2015;35(10):910-6.

23. Latorre $\mathrm{R}$ et al. Enteroendocrine cells: a review of their role in brain-gut communication. J Neurogastroenterol Motil. 2016;28(5):620-30.

24. Cantarel BL et al. Gut microbiota in multiple sclerosis: possible influence of immunomodulators. J Investig Med. 2015;63(5):729-34.

25. Kirby TO, Ochoa-Repáraz J. The gut microbiome in multiple sclerosis: a potential therapeutic avenue. Med Sci. 2018;6(3):69.

26. Gomes-Neto JC et al. A gut pathobiont synergizes with the microbiota to instigate inflammatory disease marked by immunoreactivity against other symbionts but not itself Sci Rep. 2017;7(1):1-14.

27. Round JL, Mazmanian SK. Inducible Foxp3+ regulatory T-cell development by a commensal bacterium of the intestinal microbiota. Proc Natl Acad Sci USA. 2010;107(27):12204-9.

28. Nakamura YK et al. Gut microbial alterations associated with protection from autoimmune uveitis. Invest Ophthalmol Vis Sci. 2016:57(8):374758.

29. Allen IC. Non-inflammasome forming NLRs in inflammation and tumorigenesis. Front Immunol. 2014;5:169.

30. David LA et al. Diet rapidly and reproducibly alters the human gut microbiome. Nature. 2014;505(7484):559-63.

31. Sun J. VDR/vitamin D receptor regulates autophagic activity through ATG16L1. Autophagy. 2016;12(6):1057-

32. Borody $\mathrm{T}$ et al. Fecal microbiota transplantation (FMT) in multiple sclerosis (MS): 942. Am J Gastroenterol. 2011;106:S352.

33. Friedman-Korn $\mathrm{T}$ et al. Fecal transplantation for treatment of Clostridium difficile infection in elderly and debilitated patients. Digest Dis Sci. 2018;63(1):198-203.
34. Bamba S. Successful treatment by fecal microbiota transplantation for Japanese patients with refractory Clostridium difficile infection: a prospective case series. J Microbiol Immunol Infect. 2019;52(4):663-6.

35. D’Amico E et al. A personalized approach in progressive multiple sclerosis: the current status of disease modifying therapies (DMTs) and future perspectives. Int J Mol Sci. 2016;17(10):1725.

36. Makkawi S et al. Fecal microbiota transplantation associated with 10 years of stability in a patient with SPMS. Neurol Neuroimmuno Neuroinflam. 2018;5(4):e459.

37. Okuno $T$ et al. Role of diet, gut microbiota, and metabolism in multiple sclerosis and neuromyelitis optica. Clin Exp Neuroimmunol. 2019;10:12-9.

38. Smolders J, Damoiseaux J. Vitamin $\mathrm{D}$ as a $\mathrm{T}$-cell modulator in multiple sclerosis. Vitamins Hormones. 2011;86:401-28.

39. Handel AE et al. Smoking and multiple sclerosis: an updated metaanalysis. PloS. 2011;6(1)

40. Salzer J. Vitamin A and systemic inflammation as protective factors in multiple sclerosis. Mult Scler. 2013;19(8):1046-51.

41. Loken-Amsrud $\mathrm{K}$ et al. Impact of the environment on multiple sclerosis. Tidsskr Nor Laegeforen. 2015;135(9):856-60.

42. Rojas OL et al. Recirculating intestina IgA-producing cells regulate neuroinflammation via IL-10. Cell. 2019;176(3):610-24.

43. Bhattacharjee S, Lukiw WJ. Alzheimer's disease and the microbiome. Front Cell Neurosci. 2013;7:153.

44. Bateman RJ et al. A $y$-secretase inhibitor decreases amyloid- $\beta$ production in the central nervous system. Ann Neurol. 2009;66(1):4854.

45. Roberts KF et al. Amyloid- $\beta$ efflux from the central nervous system into the plasma. Ann Neurol. 2014;76(6):837-44.

46. Evans ML et al. Bacterial Amyloids Methods Mol Biol. 2018;1779:267-88.

47. Kowalski K, Mulak A. Brain-gutmicrobiota axis in Alzheimer's disease. J Neurogastroenterol Motil. 2019;25(1):48.

48. Akbari E et al. Effect of probiotic supplementation on cognitive function and metabolic status in Alzheimer's disease: a randomized, double-blind and controlled trial. Front Aging Neurosci. 2016;8:256.

49. Jang $\mathrm{H}$ et al. Familial Alzheimer's disease Osaka mutant ( $\triangle E 22)$ $\beta$-barrels suggest an explanation for the different $A \beta 1-40 / 42$ preferred conformational states observed by experiment. J Phys Chem B. 2013;117(39):11518-29.

50. Scheltens $P$ et al. Alzheimer's disease Lancet. 2016;388(10043):505-17.

51. Kametani F, Hasegawa M. Reconsideration of amyloid hypothesis and tau hypothesis in Alzheimer's disease. Front Neurosci. 2018;12:25.

52. Bedarf JR et al. The gut microbiome in Parkinson's disease. Nervenarzt. 2019;90(2):160-6.

53. Mulak A, Bonaz B. Brain-gutmicrobiota axis in Parkinson's disease. World J Gastroenterol. 2015;21(37):10609.

54. Bennett MC: The role of a-synuclein in neurodegenerative diseases. Pharmacol Therapeut. 2005;105(3):311-31.

55. Friling S. Generation of dopamine neurons in vivo and from embryonic stem cells in vitro. 2009. Available at: https://openarchive.ki.se/xmlui/ bitstream/handle/10616/39218/thesis. pdf?sequence $=1$ \&is Allowed=y. Last accessed: 21 April 2020

56. Oakley A et al. Individual dopaminergic neurons show raised iron levels in Parkinson disease. Neurol. 2007;68(21):1820-5

57. Vijay-Kumar M et al. Metabolic syndrome and altered gut microbiota in mice lacking Toll-like receptor 5 . Science. 2010;328(5975):228-31.

58. Tillisch K, Labus JS, "Neuroimaging the microbiome-gut-brain axis", Lyte M, Cryan (eds), Microbial Endocrinology: The Microbiota-GutBrain Axis in Health and Disease (2014), Springer: London, UK, pp.40516.

59. Fernandez-Real J-M et al. Gut microbiota interacts with brain microstructure and function. $\mathrm{J}$ Clin Endocrinol Metabol. 2015;100(12):4505-13.

60. Ong IM et al. Gut microbiome populations are associated with structure-specific changes in white matter architecture. Translation Psychiatry. 2018;8(1):1-11.

61. Bagga D et al. Probiotics drive gut microbiome triggering emotional brain signatures. Gut Microbes. 2018:9(6):486-96.

62. Bagga D et al. Influence of 4-week multi-strain probiotic administration on resting-state functional connectivity in healthy volunteers. Europe J Nutrit. 2019;58(5):1821-7.

63. He $Y$ et al. Gut microbiome and magnetic resonance spectroscopy study of subjects at ultra-high risk for psychosis may support the membrane hypothesis. European Psychiatry. 2018;53:37-45.

64. Janik $\mathrm{R}$ et al. Magnetic resonance spectroscopy reveals ora 
Lactobacillus promotion of increases in brain GABA, N-acetyl aspartate and glutamate. Neuroimage. 2016;125:988-95.

65. Blasco $\mathrm{G}$ et al. The gut metagenome changes in parallel to waist circumference, brain iron deposition, and cognitive function. J Clin Endocrinol Metabol. 2017;102(8):2962-73.

66. Shim JO. Gut microbiota in inflammatory bowel disease. Pediat Gastroenterol Hepatol Nutr. 2013;16(1):17-21.

67. Chu $\mathrm{H}$ et al. Gene-microbiota interactions contribute to the pathogenesis of inflammatory bowel disease. Science. 2016;352(6289):111620.

68. Dicksved J et al. Molecular analysis of the gut microbiota of identical twins with Crohn's disease. ISME J. 2008;2(7):716-27.

69. Honda K. TFH-IgA responses keep microbiota in check. Cell Host Microbe. 2015;17(2):144-6.

70. Hall $A B$ et al. Human genetic variation and the gut microbiome in disease. Nat Rev Genet. 2017;18(11):690.

71. Liu TC et al. LRRK2 but not ATG16L1 is associated with Paneth cell defect in Japanese Crohn's disease patients. JCl Insight. 2017;2(6):e91917.

72. Elphick DA, Mahida YR. Paneth cells: their role in innate immunity and inflammatory disease. Gut. 2005;54(12):1802-9.

73. Lauro ML et al. The effect of NOD2 on the microbiota in Crohn's disease. Current Op Biotechnol. 2016:40:97102.

74. Sidiq T et al. Nod2: a critical regulator of ileal microbiota and Crohn's disease. Front Immunol. 2016;7:367.

75. Cohen LJ et al. Genetic factors and the intestinal microbiome guide development of microbebased therapies for inflammatory bowel diseases. Gastroenterol. 2019;156(8):2174-89.

76. Imhann $\mathrm{F}$ et al. Interplay of host genetics and gut microbiota underlying the onset and clinical presentation of inflammatory bowel disease. Gut. 2018;67(1):108-19.

77. Mimee $\mathrm{M}$ et al. Microbiome therapeutics-advances and challenges. Adv Drug Deliv Rev. 2016;105:44-54.

78. Rajpoot $\mathrm{M}$ et al. Understanding the microbiome: emerging biomarkers for exploiting the microbiota for personalized medicine against cancer. Semin Cancer Biol. 2018;52(Pt 1):1-8.

79. Sharma $\vee$ et al. Recent nanotechnological interventions targeting PI3K/Akt/mTOR pathway: a focus on breast cancer. Semin Cancer Biol. 2019;59:133-46.

80. Thursby E, Juge N. Introduction to the human gut microbiota. Biochem J. 2017;474(11):1823-36. 